\title{
Correction to: Trends of change in the individual contribution of risk factors for small for gestational age over more than 2 decades
}

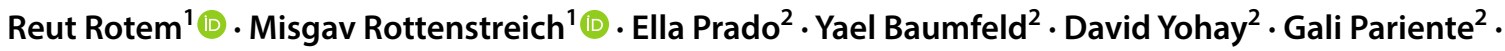 \\ Adi Y. Weintraub ${ }^{2}$
}

Published online: 14 September 2020

(c) Springer-Verlag GmbH Germany, part of Springer Nature 2020

\section{Correction to: Archives of Gynecology and Obstetrics https://doi.org/10.1007/s00404-020-05725-w}

In the original article published, the name of the corresponding author is published incorrectly. The correct name is Misgav Rottenstreich.

Publisher's Note Springer Nature remains neutral with regard to jurisdictional claims in published maps and institutional affiliations.

The original article can be found online at https://doi.org/10.1007/ s00404-020-05725-w.

\section{Misgav Rottenstreich} misgavr@gmail.com

1 Department of Obstetrics and Gynaecology, Shaare Zedek Medical Centre, Jerusalem, Affiliated with the Hebrew University Medical School of Jerusalem, Jerusalem, Israel

2 Department of Obstetrics and Gynaecology, Faculty of Health Sciences, Soroka University Medical Centre, Ben-Gurion University of the Negev, Beer-Sheva, Israel 